“@ 2018 IEEE. Personal use of this material is permitted. Permission from IEEE must be obtained for all other uses, in any current or future media, including reprinting/republishing this material for advertising or promotional purposes, creating new collective works, for resale or redistribution to servers or lists, or reuse of any copyrighted component of this work in other works." 


\title{
A Wideband Base Station Antenna with Reduced Beam Squint
}

\author{
Haihan Sun, Can Ding, and Y. Jay Guo \\ Global Big Data Technologies Centre, University of Technology Sydney, Sydney, Australia, Haihan.Sun@student.uts.edu.au
}

\begin{abstract}
This paper presents the design procedure, theoretical analysis, and experimental results of a novel wideband dual-polarized base station antenna. The proposed antenna consists of four electric folded dipoles arranged in an octagon shape that are excited simultaneously for each polarization. It provides the $\pm 45^{\circ}$ slant-polarized radiation that meets all the required specifications for base station antenna elements. Experimental results show that the proposed dualpolarized antenna has a wide bandwidth of $46.4 \%$ from 1.69 GHz to $2.71 \mathrm{GHz}$ with $\geq 15 \mathrm{~dB}$ return loss. Across this wide bandwidth, the variations of the half-power-beamwidths (HPBWs) of the two polarizations are all within $66.5^{\circ} \pm 5.5^{\circ}$, port-to-port isolation is $>30 \mathrm{~dB}$, the cross-polarization discrimination (XPD) is $>20 \mathrm{~dB}$ and, most importantly, the beam squint is $<4^{\circ}$ with a maximum $10^{\circ}$ down-tilt.
\end{abstract}

Index Terms - base station antenna, dual-polarization, stable radiation patterns, beam squint.

\section{INTRODUCTION}

The rising cost and environmental pressure in acquiring new sites for base stations and the pressure to increase capacity by mobile network operators have led to ever more stringent requirements on base station antennas. These include wide bandwidth, high port-to-port isolation, high cross-polarization discrimination (XPD), beamwidth consistency, and minimum squint. There are many challenges in meeting all these specifications simultaneously. As a result, we have witnessed rapidly increasing research activities in base station antennas in recent years [1]-[8].

Among all the base station antennas employed in the mobile industry, cross-dipole is the most commonly used type of antennas. It employs two electric dipoles placed perpendicular to each other to achieve the two polarizations [1]-[5]. Broad impedance bandwidths with excellent matching can be achieved by utilizing strong mutual coupling between the two electric dipoles. However, for a given polarization, the beamwidth of the radiation pattern from one dipole intrinsically vary significantly across a wide band [1]. In order to stabilize the radiation pattern, shaped ground [2], [3] or walls [4], [5] are employed. These techniques are suitable for single band antennas but they may cause undesirable scattering in the other band in dual band antenna systems and therefore should be avoided. Moreover, adding walls increases the size, weight, and cost of the antennas. This problem will add complexity when the antenna element is applied in a $5 \mathrm{G}$ massive MIMO system consisting of a large number of antenna elements. Recently, another configuration with four end-to-end electric dipoles are proposed in [6]-[8], which also has the potential to fulfill the specifications of the base station antenna element. Beam consistency has been achieved with half-power beamwidth (HPBW) variation $<10^{\circ}$, but the bandwidth is limited ( $<$ $30 \%$ ) in the reported works. Moreover, there has been no report on the working mechanism of such antennas. Without the insight of how this type of antennas works, it would be difficult to improve its performance.

Beam squint is one of the most crucial parameters for the down-tilted base station antenna arrays but it has rarely been discussed in the open literature. It is a parameter that measures the difference between the mechanical boresight and the horizontal beam pointing angle of the antenna, representing the symmetry of the main beam. Elliptical beamshape of an array element is the main source of squint. As an example, Fig. 1 shows the contour plot of the radiation pattern of a typical type-1 cross-dipole. Without down-tilt, the beam peak (M1) remains in the direction of the mechanical boresight. With a down-tilt angle of $10^{\circ}$, the beam peak (M2) shifts away from the mechanical boresight, thus resulting in squint. In cellular industry, the beam squint of base station antenna array is typically required to be less than $5^{\circ}$ for a down-tilt range of $0-10^{\circ}$.

In this paper, a dual-polarized antenna comprising four folded dipoles with superior performance that meets all the specifications of $4 \mathrm{G}$ base station antenna elements is proposed. Theoretical analysis is conducted based on the current distribution to obtain insights and to provide qualitative explanations to the antenna's radiation property. Experimental results that show stable radiation patterns and reduced beam squints are presented to verify the superior performance of the proposed antenna.

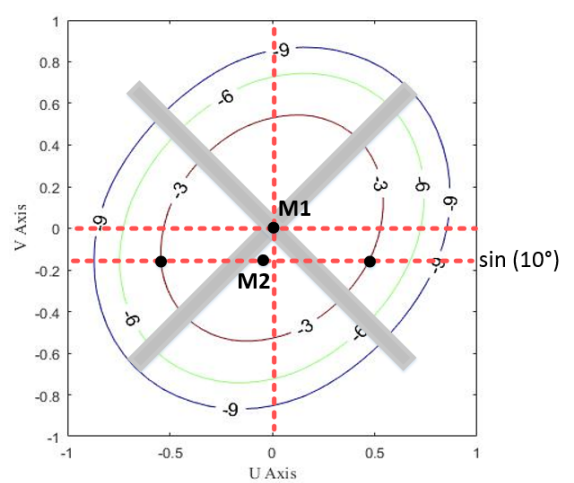

Fig. 1 Contour of the normalized radiation pattern of a cross-dipole placed above a ground plane. 


\section{ANTENNA CONFIGURATION}

The configuration of the proposed antenna is shown in Fig. 2. It consists of four folded dipoles as radiators, two microstrip baluns and a flat square reflector. The four folded dipoles are printed on a FR4 substrate and are closely arranged in the shape of an octagon. The folded dipole arms are linked together by parallel lines to form a close loop path. They are excited together by two baluns in the diagonal directions to realize the $\pm 45^{\circ}$ polarizations. The two baluns are mounted between the radiator and the reflector. Each balun consists of four essential parts: a link line (LL), a short line (SL), a transmission line (TL), and an open line (OL). Note that the two baluns are slightly different to avoid interference between the cross-over conductors. All the substrates employed in Fig. 2 are FR4 substrate with a dielectric constant of 4.3. The thickness of substrate for the radiator and baluns are $0.5 \mathrm{~mm}$ and $1.0 \mathrm{~mm}$, respectively.

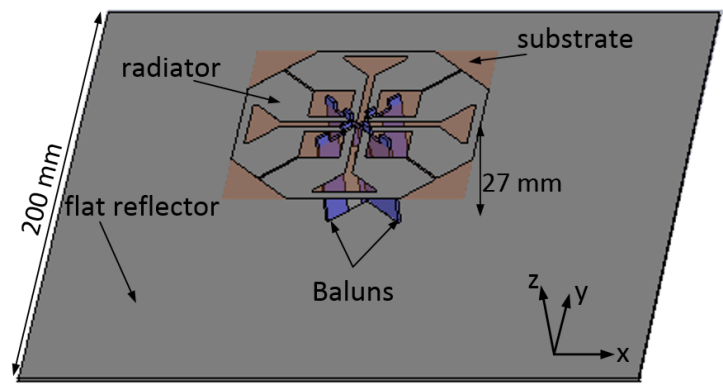

(a)

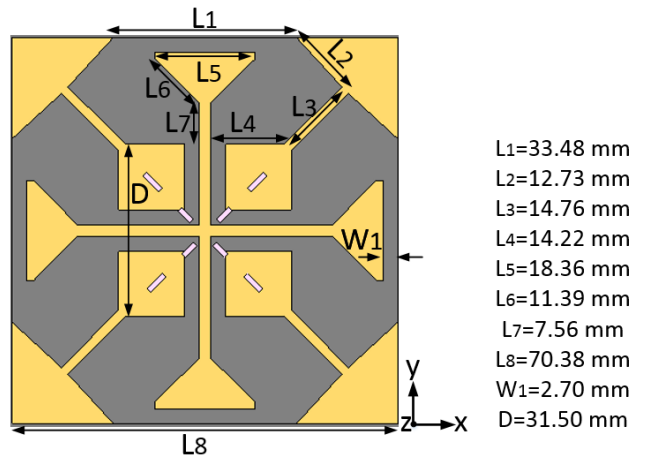

(b)
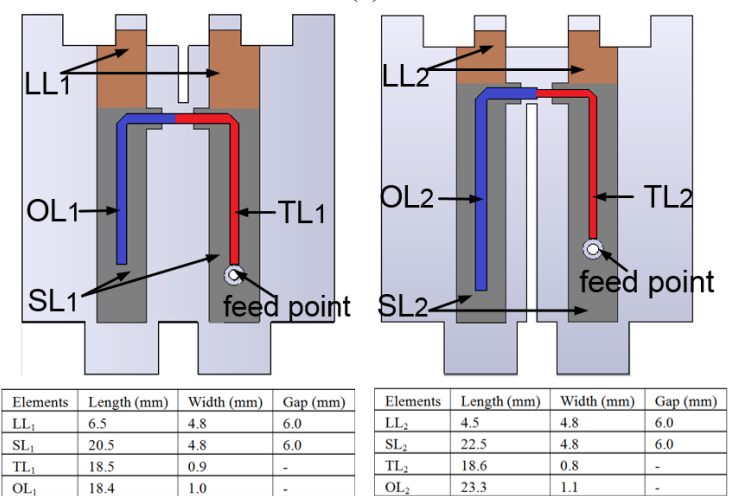

(c)

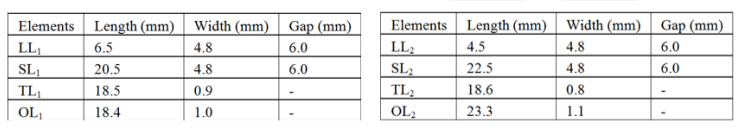

Fig. 2 (a) Perspective view of the proposed antenna, (b) top view of the aperture, and (c) perspective view of the two baluns.

\section{ANALysis of The Proposed ANTENNA}

\section{A. Half-Power Beamwidth}

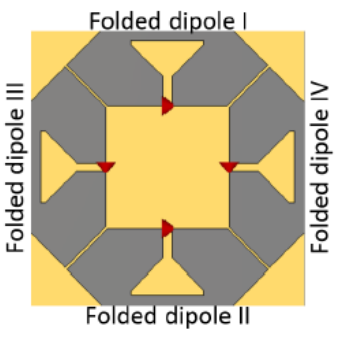

(a)

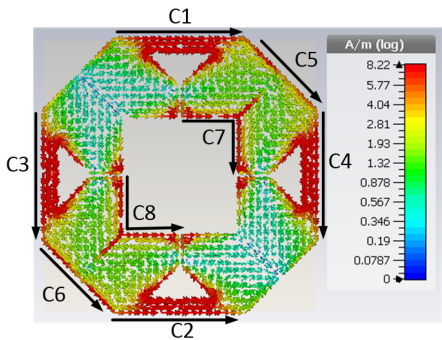

(b)
Fig. 3 (a) Configuration of the radiator without feed network, and (b) the current distribution on the radiator at $2.2 \mathrm{GHz}$.

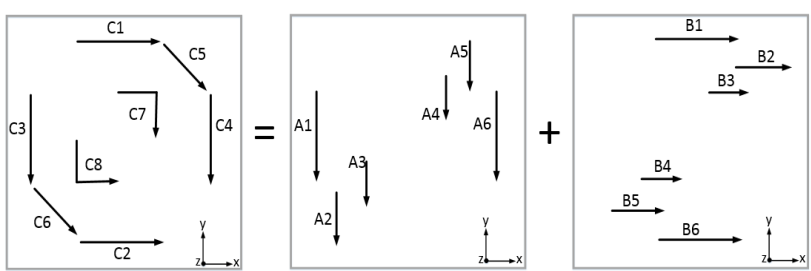

Fig. 4 Decomposed schematic current distribution at $2.2 \mathrm{GHz}$.

To understand the working principle of the proposed antenna, we examine the current distribution on four folded dipoles arranged in an octagon but without the feed networks as shown in Fig. 3(a). The folded dipoles are identical to each other and resonate at $2.2 \mathrm{GHz}$. They are designed to be excited simultaneously to realize the $\pm 45^{\circ}$ polarizations. Fig. 3 (a) shows the ideal excitation method to achieve the $-45^{\circ}$ polarization as an illustration, where the red arrows represent how the voltages are applied to the four folded dipoles. The resultant current distribution at $2.2 \mathrm{GHz}$ in this case is shown in Fig. 3 (b). It is clearly seen that there are eight dominate dipole-type currents distributed on the structure labeled $\mathrm{C} 1$ to C8. The radiation pattern of the antenna can be calculated from these currents. Owing to the diagonal symmetry, the magnitudes of the currents have the following relations:

$$
\begin{aligned}
|\mathrm{C} 1|=|\mathrm{C} 2| & =|\mathrm{C} 3|=|\mathrm{C} 4| \\
|\mathrm{C} 5| & =|\mathrm{C} 6| \\
|\mathrm{C} 7| & =|\mathrm{C} 8|
\end{aligned}
$$

Fig. 4 shows a schematic diagram of the current distribution. The currents are then decomposed into the $x$ and $y$-aligned components for the ease of analysis. We are mainly concerned with the beam consistency in the horizontal plane (xoz-plane). The H-plane pattern of the $y$ aligned currents (A1 to $\mathrm{A} 6$ ) and the E-plane pattern of the $x$ aligned currents (B1 to B6) add up and form the total radiation pattern in the horizontal plane. For a straight current placed above a reflector, the variation of the beamwidth with frequency in its H-plane is much more significant than that in its E-plane. Therefore, it can be concluded that the variation of the horizontal plane beamwidth is mainly attributed to the $y$-aligned currents (A1 to A6). In other words, if the H-plane radiation pattern introduced by the $y$-aligned currents is stabilized, the resultant entire pattern in the xoz-plane would be steady. 
Assuming that the current distributions of A1 to A6 are similar, the summed radiation pattern generated by these currents in the horizontal plane $(\varphi=0)$ can be calculated using the array theory as shown in below.

$$
\begin{gathered}
|f(\theta)|=\left|f_{1}(\theta)\right| \cdot|A F| \\
|A F|=\left|\sum_{i=1}^{6} m_{1 i} \cdot e^{j\left(k d_{1 i} \cos \theta+\xi_{1 i}\right)}\right|
\end{gathered}
$$

where $f_{i}(\theta)$ is the $\mathrm{H}$-plane radiation pattern generated by $\mathrm{Ai}$, $\mathrm{AF}$ is the array factor, and $m_{1 i}, d_{1 i}, \xi_{1 i}$ are the amplitude ratio, distance and the phase difference between $\mathrm{Ai}$ and $\mathrm{A} 1$, respectively, where $i=1,2, \ldots 6$. Current monitors were applied in CST STUDIO SUITE during the simulation to observe the magnitudes and phases of the currents. Based on the obtained results, the array factors at $1.7,2.2$, and $2.7 \mathrm{GHz}$ were calculated and plotted in Fig. 5(a). It is observed that the array factor becomes more directive with increasing frequency. Fig. 5(b) shows the H-plane patterns of a quarterwavelength dipole placed above a finite ground plane of the same size with that of the proposed antenna, which is an approximation of the currents A1-A6. According to the figure, the H-plane pattern of a single dipole $\left(\left|f_{1}(\theta)\right|\right)$ placed above a ground plane become broader with increasing frequency. The resultant H-plane radiation pattern generated by the $y$-aligned currents is then calculated from (1) and shown in Fig. 5(c), suggesting that it is stable with the frequency variation. Meanwhile, the E-plane radiation patterns of the $x$-aligned currents are also stable, as shown in Fig. 5(d). Therefore, stable H-plane radiation pattern of current components A1 to A6 and the stable E-plane radiation pattern of the current components B1 to B6 produce a steady radiation pattern in the horizontal plane (Fig. 5(e)). The variation of simulated HPBWs is $<10^{\circ}$ across the targeted band.

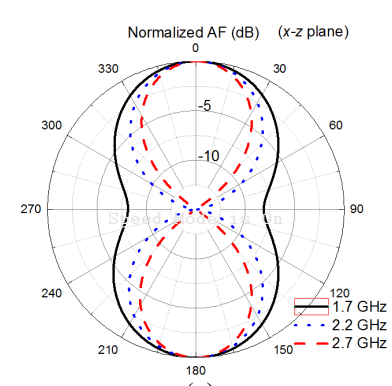

(a)

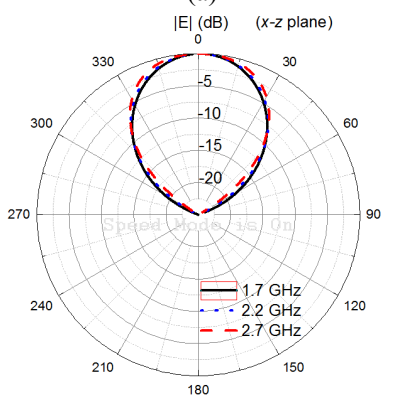

(c)

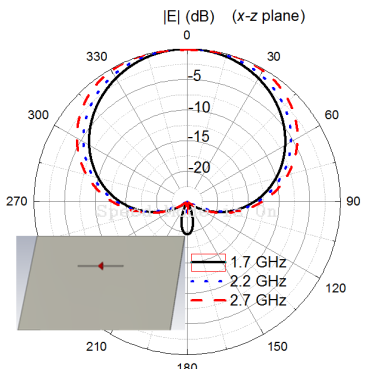

(b)

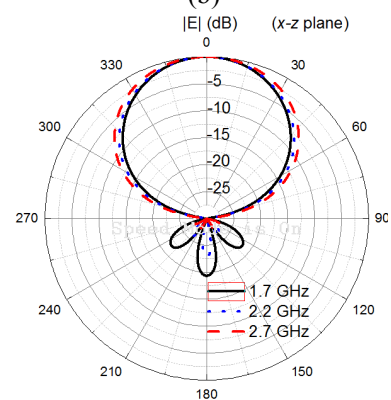

(d)

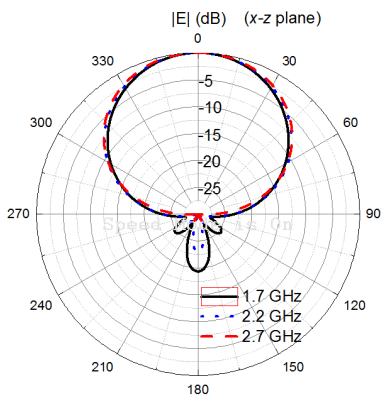

(e)

Fig. 5 (a) Calculated AF of current components A1 to A6, (b) H-plane radiation pattern of a single dipole placed above a finite ground plane, (c) calculated H-plane radiation pattern of current components A1 to A6, (d) E-plane radiation patterns resulted from B1 to B6, and (e) simulated radiation pattern of the proposed antenna in the horizontal plane at 1.7, 2.2, and $2.7 \mathrm{GHz}$.

\section{B. Beam Squint}

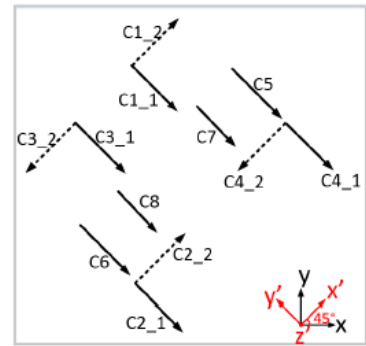

(a)

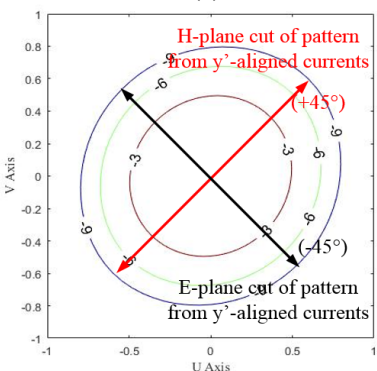

(c)

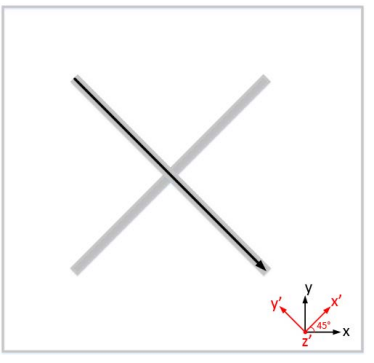

(b)

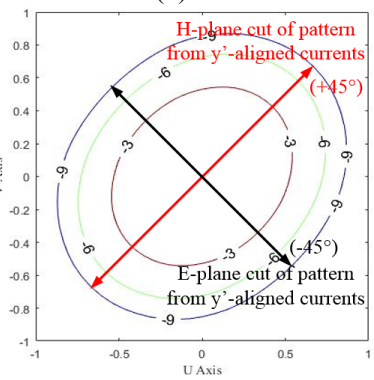

(d)
Fig. 6 (a) Decomposed abstract current distribution of the proposed antenna in the $-45^{\circ}$ and $+45^{\circ}$ directions, (b) current distribution on the cross-dipole, (c) the radiation pattern contour of the proposed antenna, and (d) the radiation pattern contour of the cross-dipole.

For $\pm 45^{\circ}$-polarized antennas, beam squint occurs when they are used in arrays with down-tilt. The long axis of the intrinsically elliptical pattern of a conventional dipole is rotated $45^{\circ}$ away from the $y$-axis, which destroys the symmetry with respect to the $y$-axis. Therefore, with a downtilt angle, the peak of the cut of our concern no longer stays at the mechanical boresight, as shown in Fig. 1. To alleviate beam squint, the solution is to make the intrinsically elliptical pattern as round as possible. To assess its ability to mitigate the beam squint, a factor $n$ is defined as $n=\mathrm{HPBW}\left(+45^{\circ}\right) / \mathrm{HPBW}\left(-45^{\circ}\right)$, which represents the ratio of HPBW in the $+45^{\circ}$ plane to that in the $-45^{\circ}$ plane. The closer the value of $n$ to 1 , the less the squint will be. Here we remark that beam squint of an antenna element is equal to 
that of the array employing the antenna elements. For the cross-dipole model shown in Fig. $1, n=1.29$ at its resonant frequency. For the proposed antenna in this paper, $n$ varies from 1.09 to 1.20 across the entire targeted band, demonstrating its ability of suppressing beam squint.

To have a better understanding, we have decomposed the currents into $x$ '- and $y$ '-aligned currents, as shown in Fig. 6 (a). It is noticed that the $x^{\prime}$-aligned currents cancelled with each other, leaving the $y^{\prime}$-aligned currents dominating the pattern. Then the target is changed into adjusting the E- and H-plane patterns of the $y$ '-aligned currents to make them identical. For a single current element, as for a typical cross dipole place above a reflector by nearly a quarter-wavelength (shown in Fig. 6 (b)), the H-plane pattern is intrinsically much wider than the E-plane pattern (shown in Fig. 6 (d)). In contrast, the proposed antenna has a more widely spread current distribution along $x$ '-axis, thereby making H-plane pattern narrower while keeping a similar E-plane pattern (shown in Fig. 6 (c)). The simulated largest beam squint is only $2.7^{\circ}$ with a maximum $10^{\circ}$ down-tilt. The beam squint problem is significantly alleviated by the proposed configuration.

To mitigate the beam squint, the strategy is to broaden the E-plane pattern and narrow the H-plane pattern of the induced current. The key point is to excite all the dipoles together and enlarge the aperture size. Therefore, the current distribution can be widened as shown in Fig. 6(a).

\section{SIMULATION AND MEASUREMENT RESULT}

A prototype was fabricated and tested. Fig. 7 plots the simulated and measured S-parameters of the two ports. The measured bandwidth for $\left|S_{11}\right| /\left|S_{22}\right| \leq-15 \mathrm{~dB}$ is $46.4 \%$ from $1.69 \mathrm{GHz}$ to $2.71 \mathrm{GHz}$, which covers the bandwidth 1.71 to $2.69 \mathrm{GHz}$ for base station application. The simulated port-toport isolation is $>35 \mathrm{~dB}$ and measured result is around $30 \mathrm{~dB}$ over the entire operation frequency band. The simulated and measured radiation patterns at $1.7,2.2$, and $2.7 \mathrm{GHz}$ in the horizontal plane are shown in Fig. 8. Only the results of $+45^{\circ}$-polarization are shown here as antenna is symmetrically arranged and the radiation patterns of the two polarizations are very similar. The simulated and measured patterns agree well with each other. The measured crosspolarization discrimination (XPD) is $>25 \mathrm{~dB}$ at the boresight and $>10 \mathrm{~dB}$ within $\pm 60^{\circ}$ of the main lobe in the horizontal plane. Fig. 9 shows the simulated and measured HPBWs of the dipole antenna for both the two polarizations. The simulated HPBWs are $64.5^{\circ} \pm 5.0^{\circ}$, and the measured HPBWs are $65.5^{\circ} \pm 4.5^{\circ}$ and $66.5^{\circ} \pm 5.5^{\circ}$ for the $+45^{\circ}$ polarization patterns and $-45^{\circ}$ polarization patterns, respectively. The measured results demonstrate that this antenna has very stable radiation patterns across a wide bandwidth. The simulated and measured gain is also shown in Fig. 9. The simulated gain varies from $9.3 \mathrm{dBi}$ to $10.1 \mathrm{dBi}$, and the measured gain varies from $9.0 \mathrm{dBi}$ to $10.7 \mathrm{dBi}$ within the targeted bandwidth. The beam squint results are shown in Fig. 10. With a maximum $10^{\circ}$ down-tilt, the simulated and measured beam squints are all $<4^{\circ}$.

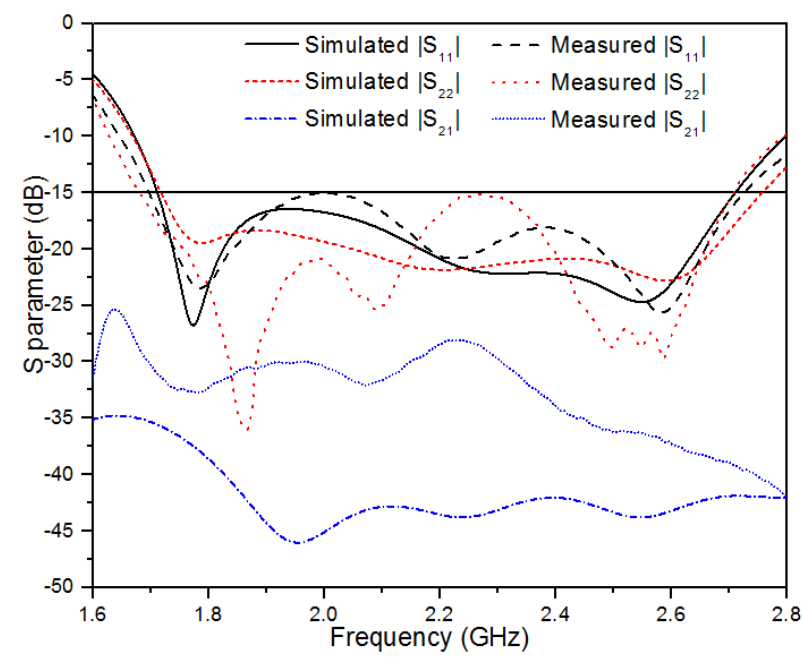

Fig. 7 Simulated and measured S-parameters

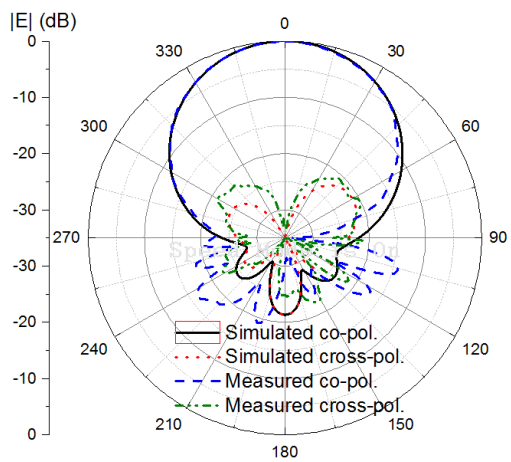

(a)

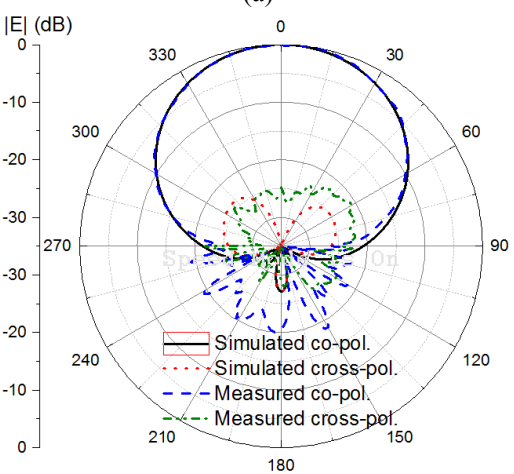

(b)

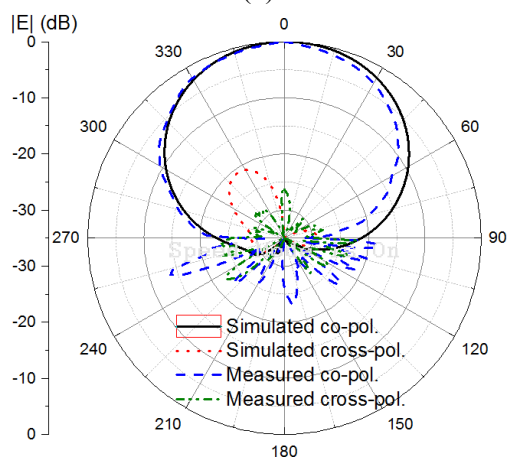

(c)

Fig. 8 Simulated and measured radiation patterns in the horizontal plane at (a) $1.7 \mathrm{GHz}$, (b) $2.2 \mathrm{GHz}$, and (c) $2.7 \mathrm{GHz}$ 


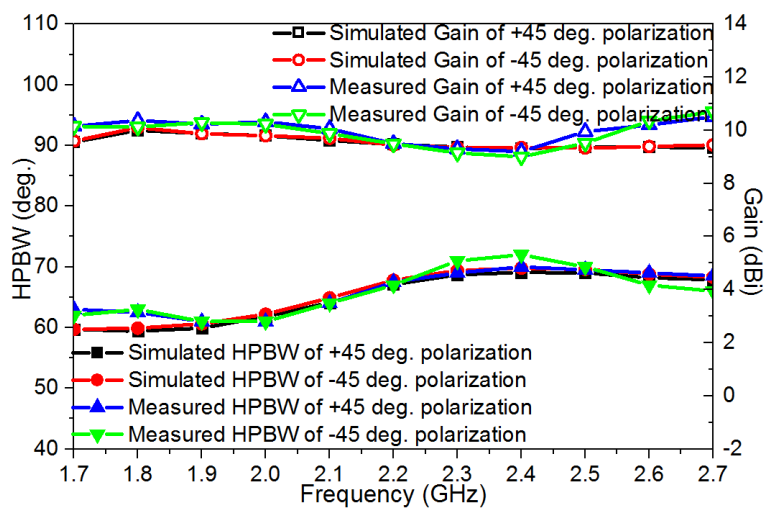

Fig. 9 Simulated and measured HPBWs and gains.

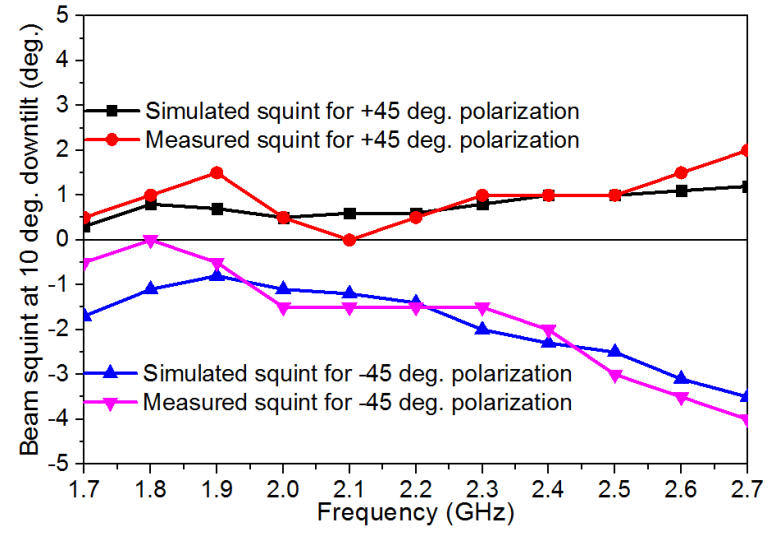

Fig. 10 Simulated and measured beam squint within $10^{\circ}$ down-tilt.

\section{CONCLUSION}

A wideband $\pm 45^{\circ}$ dual-polarized antenna utilizing four simultaneously excited folded dipoles is proposed for base station applications. The antenna features excellent radiation performance including wide bandwidth, high port-to-port isolation, low cross polarization, stable radiation patterns, and reduced beam squint. Moreover, the theoretical analysis of the radiation properties of the antenna provides a valuable guidance for the general design of base station antennas. The insight of the proposed antenna discussed in this paper can be used as a future guidance of $5 \mathrm{G}$ base station antennas.

\section{REFERENCES}

[1] Y. Gou, S. Yang, J. Li, and Z. Nie, "A compact dual-polarized printed dipole antenna with high isolation for wideband base station applications," IEEE Trans. Antennas Propag., vol. 62, no. 8, pp. 43924395, Aug. 2014.

[2] Z. Bao, Z. Nie, and X. Zong, "A novel broadband dual-polarization antenna utilizing strong mutual coupling," IEEE Trans. Antennas Propag., vol. 62, no. 1, pp. 450-454, Jan. 2014.

[3] Y. Cui, R. Li, and H. Fu, "A broadband dual-polarized planar antenna for 2G/3G/LTE base stations," IEEE Trans. Antennas Propag., vol. 62, no. 9, pp. 4836-4840, Sept. 2014.

[4] Y. Liu, H. Yi, F. W. Wang and S. X. Gong, "A novel miniaturized broadband dual-polarized dipole antenna for base station," IEEE Antennas Wireless Propag. Lett., vol. 12, pp. 1335-1338, 2013.

[5] Q. X. Chu, D. L. Wen, and Y. Luo, "A broadband $\pm 45^{\circ}$ dual-polarized antenna with Y-shaped feeding lines," IEEE Trans. Antennas Propag., vol. 63, no. 2, pp. 483-490, Feb. 2015.

[6] Y. H. Huang, Q. Wu, and Q. Z. Liu, "Broadband dual-polarized antenna with high isolation for wireless communication," Electron. Lett., vol. 45, no. 14, pp. 714-715, July 2009.
[7] D. L. Wen, D. Z. Zheng, and Q. X. Chu, "A dual-polarized planar antenna using four folded dipoles and its array for base stations," IEEE Trans. Antennas Propag., vol. 64, no. 12, pp. 5536-5542, Dec. 2016.

[8] S. G. Zhou, P. K. Tan, and T. H. Chio, "Low-profile, wideband dualpolarized antenna with high isolation and low cross polarization," IEEE Antennas Wireless Propag. Lett., vol. 11, pp. 1032-1035, 2012. 